\title{
The impact of bZIP Atf1ortholog global regulators in fungi
}

\author{
Éva Leiter ${ }^{1}$ (D) $\cdot$ Tamás Emri $^{1} \cdot$ Klaudia Pákozdi $^{1} \cdot$ László Hornok $^{2} \cdot$ István Pócsi $^{1}$ \\ Received: 8 April 2021 / Revised: 22 June 2021 / Accepted: 24 June 2021 / Published online: 24 July 2021 \\ (C) The Author(s) 2021
}

\begin{abstract}
Regulation of signal transduction pathways is crucial for the maintenance of cellular homeostasis and organismal development in fungi. Transcription factors are key elements of this regulatory network. The basic-region leucine zipper (bZIP) domain of the bZIP-type transcription factors is responsible for DNA binding while their leucine zipper structural motifs are suitable for dimerization with each other facilitiating the formation of homodimeric or heterodimeric bZIP proteins. This review highlights recent knowledge on the function of fungal orthologs of the Schizosaccharomyces pombe Atf1, Aspergillus nidulans AtfA, and Fusarium verticillioides FvAtfA, bZIP-type transcription factors with a special focus on pathogenic species. We demonstrate that fungal Atfl-AtfA-FvAtfA orthologs play an important role in vegetative growth, sexual and asexual development, stress response, secondary metabolite production, and virulence both in human pathogens, including Aspergillus fumigatus, Mucor circinelloides, Penicillium marneffei, and Cryptococcus neoformans and plant pathogens, like Fusarium ssp., Magnaporthe oryzae, Claviceps purpurea, Botrytis cinerea, and Verticillium dahliae.
\end{abstract}

\section{Key points}

- Atf1 orthologs play crucial role in the growth and development of fungi.

- Atf1 orthologs orchestrate environmental stress response of fungi.

- Secondary metabolite production and virulence are coordinated by Atfl orthologs.

Keywords bZIP-type transcription factor - Vegetative growth · Development - Environmental stress regulatory network . Secondary metabolism $\cdot$ Virulence

\section{Introduction}

Basic-region leucine zipper (bZIP)-type transcription factor proteins can form either homodimers or heterodimers with other bZIP-type transcription factors. Their DNA binding basic motifs interact with cAMP response elements (CREs) present in a number of promoters and, as a result, they control and organize a wide array of cellular processes. In fact, bZIP-type transcription factors represent one of the most ancient tran-

Éva Leiter

leiter.eva@science.unideb.hu

1 Department of Molecular Biotechnology and Microbiology, Institute of Biotechnology, University of Debrecen, P.O. Box 63, Debrecen H-4010, Hungary

2 Hungarian University of Agriculture and Life Sciences, Gödöllö, Hungary scription factor families evolved from a single eukaryotic gene. They contribute to a complex regulatory network, which plays a pivotal role in differentiation and maintenance of cells as well as orchestration of stress responses in multicellular eukaryotes (Jindrich and Degnan 2016). In plants, bZIP transcription factors (e.g., AtbZIP1 in Arabidopsis thaliana and BZI-1 in Nicotiana tabacum) are involved in development (e.g., seed induction), as well as in responses to both abiotic (e.g., drought, cold, salinity, high temperature) and biotic (i.e., herbivores, insects, and microbial pathogens) stresses (Alves et al. 2013). In humans, the bZIP-type activating transcription factor ATF2 (activating transcription factor 2) coordinates cellular homeostasis and normal organismal development, and is also involved in pathological processes (Watson et al. 2017). ATF2 regulates the expression of key genes involved in inflammatory processes, cell cycle, glycosylation, and responses to amino acid limitations (Watson et al. 2017). Unfortunately, various disorders are also associated with altered ATF2 expression and/or its phosphorylation, and 
ATF2-coupled pathological processes may affect, among others the kidneys, the nervous system, and the pancreas and may contribute to the development of different types of cancer (Watson et al. 2017).

The orthologs of ATF2 have also been identified in the genomes of fungi starting with Atf1, the first ATF2 orthologous protein described in Schizosaccharomyces pombe. Atf1 is involved in stress response, sexual development (Takeda et al. 1995), and meiotic hot spot activation (Kon et al. 1998; Hirota et al. 2007) of the fission yeast. It easily forms heterodimers with Pcrl, another bZIP-type transcription factor, but the significance of this process seems to be unequal in stress response and mating with preference for the latter in fission yeast (Kon et al. 1998; Sansó et al. 2008). According to DNA microarray-based transcriptome analyses performed in S. pombe, a core environmental stress response (CESR) mounted to oxidative, osmotic, heavy metal, alkylating, and heat stresses exists in this yeast. CESR is controlled mainly by Sty1 (Spc1) mitogen-activeted protein kinase (MAPK) and, to some extent, by Atf1 acting downstream of Sty1 (Gasch 2007; Chen et al. 2003). It is worth mentioning that there are stress response genes, whose regulation seems to be Atfl independent in S. pombe but their expression is controlled predominantly via mRNA turnover (Marguerat et al. 2014).

Several studies demonstrated that Atf1 expression is controlled both transcriptionally and posttranscriptionally in stress-exposed S. pombe. Leong et al. (2014) described a non-coding RNA locus (SPNCRNA.1164) that positively controls both atfl expression and oxidative stress response. In the fission yeast, expression levels of the oxidative stress response bZIP transcription factors, Atf1 and Pcr1, increase under $\mathrm{H}_{2} \mathrm{O}_{2}$ stress contributing to the successful adaptation of this fungus to oxidative stress. Interestingly, atfl and pcrl mRNAs contain a biased number of AAA versus AAG codons, both coding for lysine. Since the RNA polymerase II-interacting elongation complex also has an impact on the modification of some tRNAs (e.g., at the uridine wobble nucleosides), it may modulate translation efficiency in this way as well. Not surprisingly, when the histone acetyl transferase Sin3/Elp3 localized in the Elongation complex was impaired, the AAA codons were insufficiently translated resulting in reduced atf 1 and $p c r 1$ expressions and as a consequence oxidative stress-sensitive phenotypes (Fernández-Vázquez et al. 2013).

Atf1 itself controls the expression of stress-responsive genes in different manners. For example, it recruits Set1, the catalytic subunit of the highly conserved Set1C/COMPASS complex responsible for histone $\mathrm{H} 3 \mathrm{~K} 4$ methylation (H3K4me) and sets the stress-responsive and developmental genes in "poised" off state to facilitate their rapid expression, if necessary (Lorenz et al. 2014). The Sty1 (Spc1) MAPK-activated Atf1-Pcr1 bZIP heterodimer binds to oxidative stress-responsive RNAs containing the M26 sequence motif (5'-UGACGU-3' or 5'-ACGUCA-3') in addition to CRE-like DNA sites ( $5^{\prime}$-ATGACGT- $3^{\prime}$ ) and, in this manner, facilitates the stress-induced decay of these ribonucleic acids (Gao et al. 2013). It is worth mentioning that the 5'-ATGA CGT-3' sequence is also essential for M26 meiotic hot spot activation in the ade6 gene (Kon et al. 1998; Steiner et al. 2002; Hirota et al. 2007).

The genome of Saccharomyces cerevisiae also encompasses Atf-like proteins (Takeda et al. 1995), but fungi summarized in this review are closer to $S$. pombe if we consider their stress response system including the Atfl ortholog regulatory network (Miskei et al. 2009).

Since the first pioneering studies published on the versatile developmental and physiological functions of the fission yeast's Atf1, mycologists have successfully elucidated the outstanding role played by Atfl orthologs in other fungal taxa including the saprophytic model organisms, Aspergillus nidulans and Neurospora crassa, the opportunistic human parasitic fungus Aspergillus fumigatus, and plant pathonenic fungi including Fusarium graminearum and Fusarium verticillioides. In this review, we aim to focus on the diverse and complex developmental and physiological functions of the Atf1-type transcription factors in the Kingdom of Fungi.

\section{Atf1-AtfA-FvAtfA orthologs regulate vegetative growth and development}

The versatile involvements of Atf1 (S. pombe)-AtfA (A. nidulans)-FvAtfA ( $F$. verticillioides) orthologs in maintenance of vegetative growth and regulation of asexual and sexual development of fungi are summarized in Figure 1.

In. S. pombe, the lack of atfl resulted in reduced colony size at $30^{\circ} \mathrm{C}$ while no colony growth was observed at lower temparetures (Takeda et al. 1995). The $\Delta a t f l$ strain failed to mate with otherwise compatible partners indicating the crucial role of this transcription factor in the sexual development of S. pombe (Takeda et al. 1995; Shiozaki and Russell 1996).

Orthologs of $S$. pombe atfl are of outstanding importance in the coordination of growth as well as the formation of asexual and sexual spores in filamentous fungi including A. nidulans and N. crassa (Hagiwara et al. 2016; Colot et al. 2006). In A. nidulans, deletion of atfA affected vegetative growth and conidiospore formation (Balázs et al. 2010; Emri et al. 2015). The lack of atfA decreased the expression of a number of genes involved in conidiophore development as revealed by microarray-based transcriptome analysis (Emri et al. 2015; Antal et al. 2020) confirming the observation that $\triangle a t f A$ mutants showed moderate growth and produced less conidia than the control strains (Balázs et al. 2010; Emri et al. 2015). In $N$. crassa, the other widely used model filamentous fungus and ascospores of the atfl ortholog asl-1 gene deletion mutant were unable to germinate indicating the pivotal 


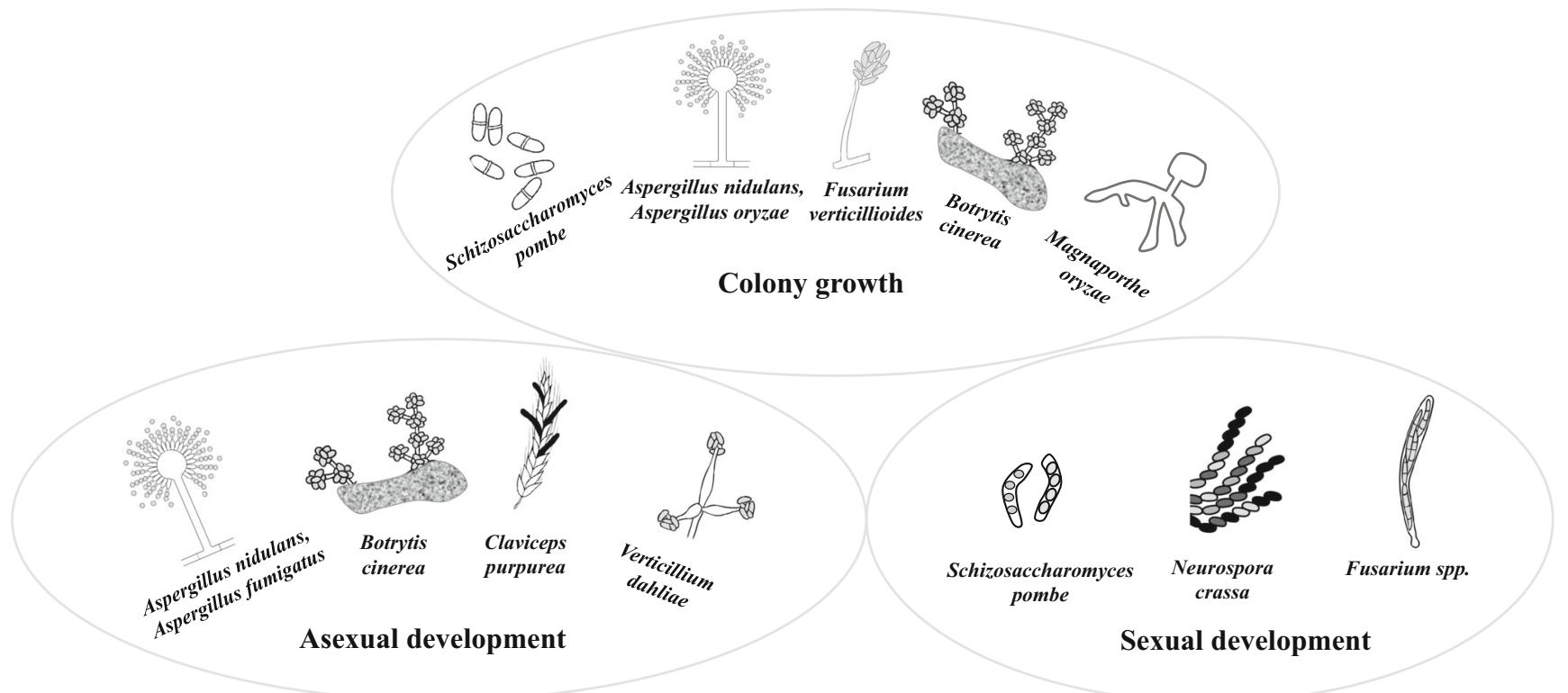

Fig. 1 Atf1-AtfA-FvAtfA orthologs are involved in the maintenance of vegetative gowth as well as in asexual and sexual developments of fungi. Schematic vegetative, asexual, and sexual morphological forms of fungi with successful functional characterization of Atf1-AtfA-FvAtfA orthologs are presented

M. circinelloides by contributing to the growth and development of the fungus during macrophage interaction as well as to the germination of spores inside the macrophages (Pérez-Arques et al. 2019). Interestingly, in another human pathogenic fungus, Penicillium marneffei, deletion of atfA caused no harm of vegetative growth or asexual development (Nimmanee et al. 2014).

Large sets of phenotypic data are also available for atf1-atfA orthologous gene deletion strains of various plant pathogenic fungi. Developmental phenotypes were observed in fusaria, e.g., deletion of Fgatfl led to delayed perithecia formation with viable ascospores in $F$. graminearum (Nguyen et al. 2013). In another study with $F$. graminearum, the role of Fgatfl in sexual reproduction was also confirmed, as the deletion mutant showed delayed ascospore release (Jiang et al. 2015). In the $\Delta$ Fgatfl $\triangle F g S K N 7$ (SKN7 encodes a nuclear response regulator and transcription factor in baker's yeast) double deletion mutant, asci accomodated eight small, single-celled ascospores instead of the typical four-celled ascospores (Jiang et al. 2015). The same double gene deletion also disturbed the asexual sporulation of $F$. graminearum resulting in smaller conidia in comparison to the wild-type strain. In Fusarium oxysporum f. sp. cubense, Foatf1 had no role in regulation of mycelial growth, but its deletion led to the formation of smaller conidia with dramatically reduced germination rates in comparison to the wild-type strain (Qi et al. 2013). In F. verticillioides, the $\triangle F$ vatfA mutant exhibited decreased vegetative growth and reduced conidium production; furthermore, conidia of the mutant were significantly smaller than that of the wild type. Unlike to the aspergilli, viability of the mutant conidia was Both genes are important elements of the virulence of 
similar to that of the wild type (Szabó et al. 2020). Reduced vegetative growth was also described in the MoAtfl deletion mutant of Magnaporthe oryzae with sparse aerial hyphae emerging on complex medium (Guo et al. 2010). Developmental defects of the $\Delta$ MoAtf1 mutant also arose in the formation of infectious hyphae since appressoria showed impaired phenotype with swollen and dark colored shortened hyphae (Guo et al. 2010).

Interestingly, the formation of sclerotia was damaged in the $\Delta$ cptfl (cptfl is orthologous to atfl) mutant of the ergot fungus Claviceps purpurea (Nathues et al. 2004). In the grey mold fungus, Botrytis cinerea, the $\Delta$ bcatf 1 mutant showed defects in both conidia production and sclerotia formation demonstrating that BcAtf1 was involved in the asexual development of this pathogenic fungus (Temme et al. 2012). BcAtfl also plays an important role in the vegetative growth of hyphae because the lack of the bcatfl gene stimulated the robust growth of aerial hyphae manifesting in a fluffy phenotype (Temme et al. 2012), which could also be observed during plant surface colonization. As showed by microarray data, expression of genes with functions in cell wall biogenesis and plant cell wall degradation was disturbed. Nevertheless, the germination rates of conidia of the wild-type and mutant strains were similar (Temme et al. 2012). In Verticillium dahliae, deletion of the ATF ortholog VDAG_08676 resulted in significantly less conidia without any impact on microsclerotia formation (Fang et al. 2017).

\section{Atf1-AtfA-FvAtfA orthologs coordinate a broad spectrum of stress responses}

bZIP-type transcription factors are crucially important in the orchestration of the environmental stress response of fungi (Chen et al. 2003; Emri et al. 2015; Rodrigues-Pousada et al. 2010) (Figure 2, Table 1). Not surprisingly, the lack of atf1 resulted in osmotic stress sensitivity when $S$. pombe was exposed to high osmolarity stress in the presence of $\mathrm{KCl}$ (Shiozaki and Russell 1996). The survival rate of the $\Delta a t f 1$ mutant after heat and oxidative stresses was also significantly lower than that of the wild-type strain (Sansó et al. 2008).

In functional genomic studies in filamentous fungi, the ascomycetous fungi A. nidulans and $N$. crassa are the most favored model organisms. In A. nidulans, deletion of atfA negatively affected the oxidative stress (e.g., $t \mathrm{BOOH}$-tert-butyl-hidroperoxide and MSB-menadione sodium bisulfite) tolerance of vegetative structures (Balázs et al. 2010; Hagiwara et al. 2008; Lara-Rojas et al. 2011; Emri et al. 2015). In addition, conidia of the $\triangle a t f A$ mutant showed increased sensitivity to oxidative, heat, osmotic, and fungicide stresses (Hagiwara et al. 2008, 2009). Hagiwara et al. (2008) and Balázs et al. (2010) also observed dramatic decrease in viability after long-term storage at $4{ }^{\circ} \mathrm{C}$ in the $\triangle$ atf $A$ mutant.
Furthermore, conidia of the gene deletion strain showed increased heat sensitivity at $50{ }^{\circ} \mathrm{C}$ after 10 - and 20 -min incubation (Hagiwara et al. 2008).

In $N$. crassa, atf- 1 is important in the response to osmotic stress and fludioxonil. Without any observable growth difference between the wild type strain and the $\Delta$ atf- 1 mutant, several genes involved in osmotic and fludioxonil stresses, i.e., cat-1 (conidia-specific catalase gene), bli-3 (bluelight-inducible gene), and $c c g-1$ (clock-controlled gene), were downregulated in the gene deletion strain (Yamashita et al. 2008). In addition, catalase (CAT1) enzyme activity was abolished in the conidia of the $\Delta a t f-1$ mutant indicating that ATF-1 controls cat- 1 gene expression during both conidiation and osmoadaptation. Nevertheless, catalase-peroxidase (CAT2) activities were similar to activities observed in the wild-type strain (Yamashita et al. 2008).

In A. oryzae, conidia of the $\triangle a t f A$ mutant were hypersensitive to $\mathrm{H}_{2} \mathrm{O}_{2}$ resulting in inhibition of conidial germination (Sakamoto et al. 2009). According to microarray analysis of global gene expression changes, a number of genes involved in oxidative stress responses were repressed in the $\triangle$ atf $A$ strain, including catalase (including catA and a $S$. cerevisiae CTA1 homolog), trehalose-6-phosphate synthase, thioredoxin, glutathione S-transferase, $\gamma$-glutamylcystein synthase, and heat shock protein genes (Sakamoto et al. 2009). Some stress-responsive genes were also under the control of another bZIP-type transcription factor, AtfB, but AtfA influenced the expression of a lot more genes (Sakamoto et al. 2009) similarly to the asymmetry observed within the Atf1-Pcr1 transcription factor pair in S. pombe. In A. oryzae, the bZIP transcription factors tested (as indicated in parentheses) affected the trehalose and mannitol content of conidiospores (AtfA and AtfB), the biosynthesis of glycerol during conidial germination (AtfA), and glutamate accumulation in the spores (AtfA) (Sakamoto et al. 2009). Deletion of atf $B$ also increased the oxidative stress sensitivity of $A$. oryzae conidia but less markedly than observed after the elimination of atfA (Sakamoto et al. 2008).

Stress-sensitive phenotypes have also been described for A. fumigatus (Hagiwara et al. 2014). After 14-day incubation at $4{ }^{\circ} \mathrm{C}$ or dessication under vacuum, conidiospores of the $\triangle a t f A$ mutant were unable to germinate underlying the central role of atfA in the maintenance of conidial viability. Regulation of some important stress-related genes was AtfA dependent in A. fumigatus conidia, including catA (catalase A), $\operatorname{dprA}$ (dehydrin-like protein), scf1 (heat shock protein), and conJ (conidiation-specific Con10 protein), and the mutant spores contained less trehalose (Hagiwara et al. 2014). In further studies by Silva et al. $(2016,2021)$, the $\triangle$ atfA strain was also sensitive to heat, oxidative (MSB, paraquat, $t \mathrm{BOOH}$, $\mathrm{H}_{2} \mathrm{O}_{2}$ ), osmotic (1.2 M sorbitol), and cell wall (Congo Red, calcofluor white) stresses, as well as to iprodione, fluodioxonil, and caspofungin $(0.5$ to $1.0 \mu \mathrm{g} / \mathrm{ml})$. It is 
Fig. 2 Atf1-AtfA-FvAtfA orthologs coordinate environmental stress response of fungi. Typical morphological forms of fungi and major stress responses governed by Atf1AtfA-FvAtfA orthologs are presented

\section{Conidia}
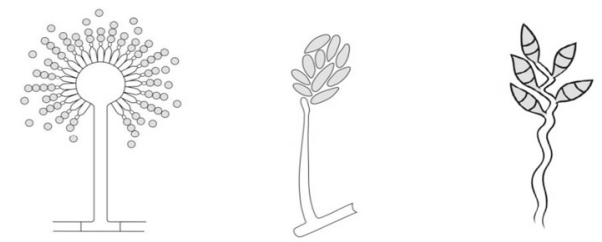

Aspergillus spp.

Fusarium spp. Magnaporthe oryzae

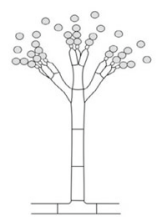

Penicillium marneffei



Verticillium dahliae

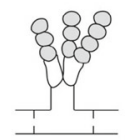

Neurospora crassa oxidative, osmotic, heat, fungicide and cell wall stresses, dessication

trehalose, mannitol, glycerol and glutamate biosyntheses and accumulations

\section{Vegetative tissue}



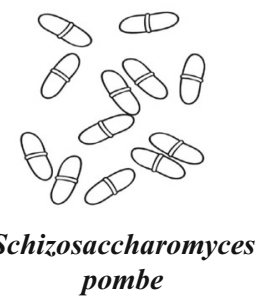

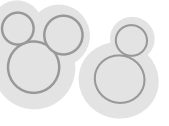

oxidative, osmotic and heat stresses noteworthy that $A$. fumigatus AtfA is also involved in the caspofungin paradoxical effect, i.e., in the elevated tolerance of high caspofungin concentrations (Valero et al. 2020). Some Atf transcription factors may also contribute to fine-tuning of the assimilation of carbon sources and the accumulation of reserves like trehalose and glycogen (Silva et al. 2021).

In P. marneffei, deletion of atfA increased the oxidative stress sensitivity of the fungus in the presence of $t \mathrm{BOOH}$ but its osmotic stress sensitivity and cell wall integrity remained unaltered (Nimmanee et al. 2014). Furthermore, inactivation of atf1 resulted in higher sensitivity to heat stress $\left(40^{\circ} \mathrm{C}\right)$ in Cryptococcus neoformans yeast cells (Kim et al. 2010).

Extensive phenotypic characterizations of atf1-atfA orthologous gene deletion mutants have also been carried out in plant pathogenic fungi including Fusarium spp., $M$. oryzae, and $V$. dahliae. As expected, a number of stress sensitivity phenotypes have been recorded for the gene deletion strains, laying emphasis on the distinguished role of these bZIP-type transcription factors in the maintenance of the stress defense systems of these successful plant pathogens.

In $F$. graminearum, the $\Delta F g a t f 1$ deletion mutant showed increased sensitivity to oxidative (in the presence of $0.5 \mathrm{mM}$ $\mathrm{H}_{2} \mathrm{O}_{2}$ ) and osmotic (tested with $\mathrm{NaCl}, \mathrm{KCl}$ and sorbitol) as well as cell wall (150 $\mu \mathrm{g} / \mathrm{ml}$ Congo Red treatment) stresses compared to the wild-type strain (Jiang et al. 2015; Nguyen et al. 2013). In F. verticillioides, FvatfA was also important in oxidative stress defense and cell wall integrity (Szabó et al. 2020). Deletion of FvatfA brought about increased sensitivity to $\mathrm{H}_{2} \mathrm{O}_{2}, \mathrm{MSB}$, and CongoRed but heat and cold sensitivity of its conidia was comparable to that of the wild-type strain (Szabó et al. 2020).

In $M$. oryzae, both mycelia and conidia of the Moatfl deletion mutant were sensitive to $\mathrm{H}_{2} \mathrm{O}_{2}$ (Guo et al. 2010), and a high number of $\mathrm{H}_{2} \mathrm{O}_{2}$-treated conidia of the mutant were unable to germinate even after 12-h cultivation as compared with the wild-type strain (Guo et al. 2010). Interestingly, although altered intracellular accumulation of reactive oxygen species (ROS) was not detected, reduced laccase and peroxidase activities were measured in the $\Delta$ Moatfl strain, which is likely to increase the vulnerability of the fungus to the ROS-mediated defense of the host plant (Guo et al. 2010).

In $V$. dahliae, deletion of the ATF ortholog VDAG_08676 gene caused no change in oxidative stress sensitivity of the fungus, but higher levels of ROS were generated in the mutant with concomitantly reduced laccase and peroxidase activities as compared to the wild type (Fang et al. 2017). 
Table 1 Atf1-AtfA-FvAtfA orthologs involved in the stress responses of fungal species

\begin{tabular}{|c|c|c|c|}
\hline Species & Orthologs & Stress & Reference \\
\hline S. pombe & Atfl & $\begin{array}{l}\text { Osmotic }(\mathrm{KCl}) \\
\text { Heat }\left(45^{\circ} \mathrm{C}\right) \\
\text { Oxidative }\left(\mathrm{H}_{2} \mathrm{O}_{2}\right)\end{array}$ & $\begin{array}{l}\text { Shiozaki and Russell 1996; Sansó et al. } \\
2008\end{array}$ \\
\hline A. nidulans & AtfA & $\begin{array}{l}\text { Oxidative ( } t \mathrm{BOOH}, \mathrm{MSB} \text {, } \\
\left.\quad \mathrm{H}_{2} \mathrm{O}_{2}\right) \\
\text { Heat }\left(50^{\circ} \mathrm{C}\right) \\
\text { Fungicide (fludioxonil) }\end{array}$ & $\begin{array}{l}\text { Balázs et al. 2010; Hagiwara et al. 2008; } \\
\text { Lara-Rojas et al. 2011; Emri et al. 2015; } \\
\text { Hagiwara et al. } 2009\end{array}$ \\
\hline N. crassa & Atf-1/Asl-1 & $\begin{array}{l}\text { Osmotic }(\mathrm{NaCl}) \\
\text { Fungicide (fludioxonil) }\end{array}$ & Yamashita et al. 2008; Colot et al. 2006 \\
\hline A. fumigatus & AtfA & $\begin{array}{l}\text { Cold }\left(4{ }^{\circ} \mathrm{C}\right) \\
\text { Dessication } \\
\text { Heat } \\
\text { Oxidative ( } t \mathrm{BOOH}, \mathrm{MSB} \text {, } \\
\mathrm{H}_{2} \mathrm{O}_{2} \text {, paraquat) } \\
\text { Osmotic (sorbitol) } \\
\text { Cell wall (Congo Red and } \\
\text { calcofluor white) } \\
\text { Fungicide (fluodioxonil and } \\
\text { caspofungin) }\end{array}$ & $\begin{array}{l}\text { Hagiwara et al. 2014; Valero et al. 2020; } \\
\text { Silva et al. 2016, } 2021\end{array}$ \\
\hline P. marneffei & AtfA & Oxidative $(t \mathrm{BOOH})$ & Nimmanee et al. 2014 \\
\hline A. oryzae & AtfA & Oxidative $\left(\mathrm{H}_{2} \mathrm{O}_{2}\right)$ & Sakamoto et al. 2009 \\
\hline F. graminearum & FgAtf1 & $\begin{array}{l}\text { Oxidative }\left(\mathrm{H}_{2} \mathrm{O}_{2}\right) \\
\text { Osmotic }(\mathrm{NaCl}, \mathrm{KCl} \text {, and } \\
\quad \text { sorbitol) } \\
\text { Cell wall (Congo Red) }\end{array}$ & Jiang et al. 2015; Nguyen et al. 2013 \\
\hline F. verticillioides & FvAtfA & $\begin{array}{l}\text { Oxidative }\left(\mathrm{H}_{2} \mathrm{O}_{2}, \mathrm{MSB}\right) \\
\text { Cell wall (Congo Red) }\end{array}$ & Szabó et al. 2020 \\
\hline M. oryzae & MoAtf1 & Oxidative $\left(\mathrm{H}_{2} \mathrm{O}_{2}\right)$ & Guo et al. 2010 \\
\hline
\end{tabular}

\section{Integration of Atf1-AtfA-FvAtfA orthologs into the fungal stress response regulatory network}

AtfA and its orthologs are regulated by stress-activated protein kinases (SAPK) in fungi. In S. pombe, Atfl is under the control of the well-known Sty1 SAPK which plays a predominant role in the regulation of genes during diverse forms of stress including heat, high osmolarity, oxidative, and starvation stresses (Kato Jr et al. 1996; Sánchez-Mir et al. 2018). Under various environmental stress conditions, Sty1 is phosphorylated, and then enters the nucleus where it binds and phosphorylates Atfl leading to upregulation of several genes including the atfl gene itself (Gaits et al. 1998; Salat-Canela et al. 2017). It is also suggested that Atfl is activated by the Pmk1 mitogen-activated protein kinase (MAPK) of the cell integrity pathway which is involved in the response to hypoor hyper-osmotic stress, oxidative stress, glucose starvation stress, or cell wall damage stress, and contributes to cytokinesis and the maintenance of ion homeostasis (Takada et al. 2007; Pérez et al. 2018). Atfl is constitutively localized in the nucleus under unstressed and nitrosative stress conditions; however, upon prolonged stress, it translocates into the cytoplasm for unknown reasons (Kar et al. 2018). Atfl upregulates most of its target genes as a heterodimer formed with Pcr1. A few genes, however, do not need Pcrl for their upregulation (Sansó et al. 2008). These genes may be upregulated by an Atf1 homodimer or by a not yet identified heterodimer of Atf1 (Sansó et al. 2008). As an example, Atf21 is postulated as a potential partner for heterodimer formation with Atfl under KCl-induced stress (Sansó et al. 2008). Atfl can interact with other transcription factors on the promoters of its target genes as well: Salat-Canela et al. (2017) suggested that Atfl and Pap1 act synergistically during the upregulation of antioxidant enzyme genes and both transcription factors should be loaded on DNA for full upregulation. Moreover, due to its chromatin remodeling activity, Atf1 downregulates the basal expression of several genes under unstressed conditions (Sansó et al. 2011). Apart from its DNA binding function, Atfl can physically bind to the anaphase-promoting complex/cyclosome (APC/C) as well and this protein-protein interaction is important in controlling cell cycle progression (Ors et al. 2009).

In A. nidulans and A. fumigatus, both activity of the AtfA protein and transcription of the atfA gene is controlled by the 
SakA (HogA) SAPK pathway under stress (Hagiwara et al. 2009; Miskei et al. 2009; Silva et al. 2017). When phosphorylated, SakA binds to AtfA in the nuclei and this step is necessary for AtfA-dependent gene activations in both species (Lara-Rojas et al. 2011; Silva et al. 2021). SakA together with AtfA contributes to oxidative, osmotic, and nutrient starvation stress responses and they are required for the proper regulation of asexual and sexual development in A. nidulans (Kawasaki et al. 2002; Lara-Rojas et al. 2011). In A. fumigatus, SakA and AtfA are important in adaptations to oxidative, osmotic, cell wall damage, and heat stresses (de Oliveira Bruder Nascimento et al. 2016). Manfiolli et al. (2019) demonstrated in A. fumigatus that SakA physically interacts with other MAPKs like the functionally redundant MpkC (de Oliveira Bruder Nascimento et al. 2016), a paralog of SakA, or MpkA (Valiante et al. 2015), a key player in the maintenance of cell wall integrity. These observations suggest that like in S. pombe (Takada et al. 2007; Pérez et al. 2018), AtfA is under the control of more than one MAPKs/SAPKs in Aspergillus species. AtfA was constitutively nuclear localized under unstressed conditions and upon stress in A. fumigatus, too (Silva et al. 2021). It contrasts with the behavior of AtfB, AtfC, and AtfD bZIP transcription factors of the same species, where the distribution between cytosol and nucleus highly depends on stress conditions, and their pronounced nuclear location was only observed under stress (Silva et al. 2021). Physical interaction between AtfA and AtfB was first suggested by Lara-Rojas et al. (2011) in A. nidulans and, more recently, it was demonstrated that besides the genetic interaction with the AtfA-D transcription factors in A. fumigatus, AtfA was also able to form heterodimers with all the other three tested bZIP transcription factors under various stress conditions (Silva et al. 2021).

Transcriptome data demonstrated that, like in the case of S. pombe Atf1, A. nidulans AtfA also regulated the basal transcription of many stress-related and stress-unrelated genes in unstressed cultures (Emri et al. 2015; Orosz et al. 2017). Genome-wide transcriptional changes detected concomitantly in a wild-type and an atfA gene deletion mutant suggested that besides its direct effects, AtfA also regulated the transcription of numerous genes indirectly during stress treatments (Orosz et al. 2017; Antal et al. 2020). It was postulated that AtfA was involved in the transcriptional upregulation of two component signal transduction system genes (including $p h k A, p h k B, f p h A$, $y p d A, t c s B)$ in $A$. nidulans based on their systemic downregulation in the atfA gene deletion mutant under versatile culturing conditions and the occurrence of putative Atf-binding sites in their promoters (Antal et al. 2020; Emri et al. 2021). Interestingly, some of the related proteins like NikA, YpdA, SskA, TcsA, and TcsB are upstream regulators of SakA in this fungus (Hagiwara et al. 2009; Miskei et al. 2009; Lara-Rojas et al. 2011). This postulated regulatory function of AtfA could modulate the activity of the signaling network upon stress leading to indirect transcriptional changes of a huge number of genes (Antal et al. 2020; Emri et al. 2021). Concurrently, deletion of A. fumigatus sakA encoding the upstream SAPK regulator of AtfA prevented the upregulation of two component signal transduction genes under stress (Silva et al. 2017).

Nevertheless, further studies are definitely needed and advisable (i) to describe the whole spectrum of genes under direct AtfA control, (ii) to shed light on the interacting partners of AtfA forming, e.g., heterodimers with it, and (iii) to reveal the interplays and cross-talks existing between AtfA and other stress response, developmental, and vegetative growth maintenance regulatory elements.

Summing it up, recent data suggest that the regulatory role of Atfl-AtfA-FvAtfA orthologs in fungi goes far beyond the upregulation of certain genes when it is necessary. They also affect the basal transcription level of genes under unstressed conditions and modulate the activity of the signaling network via physical interactions with other transcription factors and the transcriptional regulation of the signaling genes themselves upon stress.

\section{Atf1-AtfA-FvAtfA orthologs are important players in the regulation of secondary metabolism}

A growing body of experimental data indicates that the biosynthesis of secondary metabolites (SMs) is tightly coupled to the oxidative stress response of fungi. AtfB, another bZIP transcription factor, together with additional transcription factors, including AP-1, MsnA, SrrA, and possibly AtfA were found to regulate both aflatoxin biosynthesis genes and oxidative stress response genes in Aspergillus parasiticus (Hong et al. 2013a). Based on the co-regulation of SM biosynthetic and oxidative stress response genes found in a number of fungi, a theory of double defense system working against ROS-elicited damages has been proposed by several researchers (Roze et al. 2011; Yin et al. 2012; Hong et al. 2013b). In this defense system, antioxidant scavengers constitute the first line, while production of SMs forms the second line of defense. The prominent role of Atf1-AtfA-FvAtfA orthologs in response to oxidative stress as proven in a variety of fungus species indicates that these transcription factors may also be important players in the regulation of SM biosynthesis (Emri et al. 2015; Jung et al. 2016; Pérez-Arques et al. 2019; Jung et al. 2016; Nguyen et al. 2013; Temme et al. 2012; Szabó et al. 2020). However, studies on the possible direct role of Atf1-AtfA-FvAtfA-type transcription factors in the regulation of SM production, i.e., when SM producing capabilities of the appropriate gene deletion mutants and their wild-type parental strains were compared in vitro, gave diverse results (Emri et al. 2015; Jung et al. 2016; 
Pérez-Arques et al. 2019; Jung et al. 2016; Nguyen et al. 2013; Temme et al. 2012; Szabó et al. 2020).

Comparing the global gene expression profiles of an A. nidulans $\triangle a t f A$ mutant, characterized by an elevated sensitivity to oxidative $\left(\mathrm{H}_{2} \mathrm{O}_{2}, \mathrm{MSB}\right.$, tBOOH, diamide) stress with profiles of its parental wild-type strain, revealed remarkable transcriptional differences under either unstressed or stressed conditions (Emri et al. 2015). In the absence of stress, 657 and 542 genes were upregulated and downregulated, respectively, in the mutant in comparison to the wild-type strain. In subsequent stress exposure experiments, two levels of stress conditions were investigated: treatments with MSB, tBOOH, and diamide generated "severe" stress, while $\mathrm{H}_{2} \mathrm{O}_{2}$ and $\mathrm{NaCl}$ treatments caused "mild" stress. As expected, more than 1000 genes were differentially regulated in cultures subjected to "severe" stress, while "mild" stress treatments resulted in less profound global transcriptional changes (Emri et al. 2015). Importantly, stress exposures also altered the transcription of a number of putative SM genes; altogether, 155 and 112 SM genes were upregulated and downregulated, respectively, in the wild-type reference strain. On the other hand, the same stress treatments resulted in significantly enhanced gene expression changes in the $\triangle a t f A$ mutant, where 179 and 139 SM genes were upregulated and downregulated, respectively (Table 2) (Emri et al. 2015). These findings clearly demonstrated that all kinds of oxidative stressors significantly affected the transcription of SM biosynthesis genes and, most importantly, AtfA turned out to be a key player in these events.

In addition to oxidative stress, other types of environmental stresses, e.g., osmotic $(\mathrm{NaCl})$ and heavy metal $\left(\mathrm{Cd}^{2+}\right)$ stress, may also affect significantly the expression patterns of SM biosynthesis gene clusters in A. nidulans (Table 2). Importantly, $\mathrm{H}_{2} \mathrm{O}_{2}$ and $\mathrm{Cd}^{2+}$ exposures seem to be effective in the upregulation of various SM clusters in the $\triangle a t f A$ mutant (Table 2). This observation may lead to the development of novel strain improvement strategies and even new industrial SM production platforms. Nevertheless, other kinds of stress treatments $(t \mathrm{BOOH}$, diamide, $\mathrm{NaCl})$ turned out to be mostly repressive for many SM clusters in the absence of atfA and the loss of AtfA even resulted in reduced stress responsiveness for some clusters (Table 2). These findings clearly indicate the limits of this approach (deletion of atfA combined with stress treatments) to activate inactive SM biosynthetic gene clusters in the Aspergilli (Emri et al. 2015; Reverberi et al. 2008, 2012; Amare and Keller 2014).

Atfl-AtfA orthologs may play an important role in the regulation of SM biosynthesis genes in human pathogenic fungi, too. For example, the A. fumigatus GprK G protein-coupled receptor, which stimulates the transcription of atfA and sakA, was also involved in the regulation of oxidative stress response and gliotoxin biosynthesis (Jung et al. 2016). It is important to note that gliotoxin, secreted by A. fumigatus conidia, is a prerequisite of the successful invasion of host by decreasing ciliary movement of bronchial epithelial cells (Tomee and Kauffman 2000). In M. circinelloides, a $\Delta a t f 1$ mutant and its wild-type parental strain were co-cultured with mouse macrophages to evaluate the behavior of the mutant in the stressful environment present during phagocytosis (Pérez-Arques et al. 2019). In vitro hostpathogen RNA sequencing was then performed to assess the transcriptional differences between the two strains. Altogether, 3384 genes were differentially expressed in the $\Delta$ atfl mutant in comparison to the wild-type strain, among which 15 were annotated as putative SM biosynthesis genes and their vast majority (14 out of 15 ) were downregulated in the absence of functional Atf1.

The regulatory function of Atf1-AtfA-FvAtfA orthologs on SM production is especially well studied in some plant pathogenic fungi. For example, $\Delta$ Fgatfl mutants constructed in F. graminearum strain PH-1 by Nguyen et al. (2013) showed elevated tolerance to hydrogen peroxide when tested on a complete medium $(\mathrm{CM})$ supplemented with 10,15 , and $20 \mathrm{mM} \mathrm{H}_{2} \mathrm{O}_{2}$ and they also produced some 3.5 and 1.7 times more deoxynivalenol (DON), a trichothecene mycotoxin after 1 and 3 days' culturing in vitro, respectively, as compared to the wild-type strain. The increased DON production paralleled with a significant upregulation of key trichothecene biosynthesis genes (tri4, tri5, tri6, and tri10). Importantly, DON production of the $\Delta F g a t f 1$ strain was strongly reduced during infection of wheat heads. The $\Delta F$ gatfl strains that also produced higher amounts of aurofusarin, based on the intense red color of their colonies and genes (gip1, gip 2, pks12) involved in aurofusarin biosynthesis, were upregulated in the mutant. On the other hand, production of zearalenone (ZEA), a third important $\mathrm{SM}$ of $F$. graminareum as measured under in vitro conditions, was similar in the gene deletion mutants and the wild-type and transcript levels of ZEA biosynthesis genes (zeal, zeb1, zeb2) were also similar in the mutants and their parental strain.

Studies using another $\Delta F$ gatfl mutant of $F$. graminearum PH-1 gave somewhat different results suggesting that the regulatory effect of FgAtfl may diverge even at inter-strain level and the results greatly depend on experimental conditions. The gene deletion mutant of this fungus, produced by Jiang et al. (2015) showed slightly reduced growth on CM supplemented with $0.5 \mathrm{mM} \mathrm{H}_{2} \mathrm{O}_{2}$ as compared with the wild type indicating that the sensitivity of this fungus towards ROS increased in the absence of functional Atf1. Furthermore, no significant differences were observed in DON production, tested in rice grain cultures between the mutant and its parental strain suggesting that deletion of atfl has no influence on trichothecene biosynthesis.

In $F$. verticillioides, deletion of FvatfA led to dramatic changes in SM production. Concentrations of the polyketide-type mycotoxins, fumonisin B1 and B2 (FB1, FB2), potential causes of life-threatening disorders in humans 
Table 2 Secondary metabolite gene clusters affected by atfA gene deletion in Aspergillus nidulans
Cluster (number of genes in the cluster ${ }^{\mathrm{a}}$ )

Stress dependence (number of stress-responsive genes in the cluster)

Clusters become stress responsive in the absence of AtfA in A. nidulans

Monodictyphenone (mdp) cluster $(12)^{\mathrm{b}}$
Derivative of benzaldehyde1 (dba) and F9775 hybrid
$\quad$ cluster $1(9)^{\mathrm{b}}$

Upregulated in $\mathrm{H}_{2} \mathrm{O}_{2}$ stress (11)

Downregulated in $t \mathrm{BOOH}$ stress (8)

Downregulated in $\mathrm{NaCl}$ stress (8)

Upregulated in $\mathrm{H}_{2} \mathrm{O}_{2}$ stress (5)

Downregulated in $t \mathrm{BOOH}$ stress (6)

Downregulated in diamide stress (6)

Downregulated in $\mathrm{NaCl}$ stress (6)

pkf cluster $(6)^{\mathrm{b}}$

Upregulated in $\mathrm{H}_{2} \mathrm{O}_{2}$ stress (4)

Downregulated in $t \mathrm{BOOH}$ stress (4)

Downregulated in diamide stress (5)

Downregulated in $\mathrm{NaCl}$ stress (5)

ivo cluster $(2)^{\text {b,c }}$

Upregulated in $\mathrm{H}_{2} \mathrm{O}_{2}$ stress (2)

Downregulated in $t \mathrm{BOOH}$ stress (2)

Downregulated in diamide stress (2)

Downregulated in $\mathrm{NaCl}$ stress (2)

AN7884 cluster (15) ${ }^{\mathrm{d}}$

Upregulated in $\mathrm{CdCl}_{2}$ stress (7)

AN7084 cluster (9) ${ }^{\mathrm{d}}$

Upregulated in $\mathrm{CdCl}_{2}$ stress (6)

Clusters lost their stress responsiveness in the absence of AtfA in A. nidulans

Derivative of benzaldehyde1 (dba) and F9775 hybrid cluster $2(10)^{\mathrm{b}}$

AN1242 cluster (6) ${ }^{\mathrm{d}}$

Upregulated in $t \mathrm{BOOH}(6)$

Downregulated in $\mathrm{CdCl}_{2}$ stress (4)

${ }^{a}$ Only genes of clusters determined by either manually or experimentally were involved in the analysis (Inglis et al. 2013)

${ }^{\mathrm{b}}$ Only clusters where more than the half of the cluster genes including at least one key gene showing upregulated or downregulated were regarded as stress-responsive cluster. In this case, genes showing at least two-time increase or decrease in their relative transcription levels were regarded as upregulated and downregulated genes, respectively (Emri et al. 2015)

${ }^{\mathrm{c}}$ Out of the clusters containing four or less genes only the cluster where all the cluster genes showed upregulated or downregulated were regarded as stress-responsive cluster

d Only the clusters where the cluster genes were significantly enriched (Fisher's exact test, $p<0.05$ ) in the upregulated or downregulated gene sets were regarded as stress-responsive cluster. In this case, all differentially expressed genes were regarded as stress-responsive genes (Emri et al. 2021) and animals upon ingestion were below the detection limit as measured by CE-MS in the gene deletion mutant grown in Myro medium for 14 days, while the wild type produced normal amounts of these metabolites under the same conditions. Expression of two fumonisin biosynthesis genes, fuml and fum 8 , paralleled with fumonisin production: transcript levels of these two genes were significantly lower in the $\Delta F v a t f A$ mutant in comparison to the wild type. Furthermore, the gene deletion mutant produced only trace amounts of carotenoids when cultured in liquid minimal medium under continuous illumination for 7 days, whereas the wild-type synthesized normal amounts of these SMs. CarRA and $\operatorname{CarB}$, two key enzyme genes of carotenoid biosynthesis, were significantly downregulated in $\triangle F v a t f A$. In silico, promoter analyses aimed to trace the presence of the consensus sequence, TGACGTCA (known as the binding site for ATF/CREB bZIP transcription factors), on promoters of the genes found to be downregulated in the $\triangle F$ vatfA mutant which led to the identification of this motif on the sequences of fum 1, fum8, $\operatorname{car} R A$, and $\operatorname{car} B$. On the other hand, the $\triangle F v a t f A$ mutant produced approximately 10 times more bikaverin, a deep-red pigment with antimicrobial and anticancer activities than the wild type. Surprisingly, expression of bik1, a key polyketide synthase gene, responsible for bikaverin synthesis in $F$. verticillioides showed no increase in the mutant indicating that the causes of overproduction of this metabolite are others than upregulation of bikl. The most likely explanation of bikaverin overproduction is the drastic downregulation of the fumonisin and carotenoid pathways in $\triangle F v a t f A$. Due to these changes in the mutant, the building blocks needed for the production of fumonisins and 
carotenoids were probably accumulated and channelled towards the synthesis of other metabolites, including bikaverin. An interesting novelty of this research was the strongly reduced carotenoid production observed in the absence of AtfA. As mentioned before, SMs have been suggested to play a role in protection against ROS, but direct proofs of ROS-scavenging ability of most of these metabolites are lacking. Carotenoids are, however, well-documented antioxidants (Avalos and Limón 2015); their regulation by such a key player of stress mediation, like Atf1/AtfA, provides an unambiguous proof on the causal relation between ROS stress and response by SM production (Szabó et al. 2020).

Although deletion of atfl caused no change in oxidative stress sensitivity of $B$. cinerea, the $\Delta B$ catf1 mutant was found to produce higher amounts of secondary metabolites (botrydial, botryendial, and botcinin) as compared to the wild type and a number of genes coding for botrydial and botcinic acid was also upregulated in the mutant (Temme et al. 2012). Studies on the genome-wide effects of BcAtfl brought to light the exceptional importance of this TF on SM production. When total mRNA samples of $B$. cinerea wild type and its $\triangle B$ catf1 mutant grown under unstressed conditions were subjected to microarray analyses, 511 genes including 34 predicted SM-encoding ORFs were found to be differentially expressed in the mutant (Temme et al. 2012). The number of SM genes, upregulated in the mutant, was significantly higher (26) than the number of downregulated ones (8) indicating that Atf1 exerts a repressing effect on the majority of SM genes at least in this fungus and under culture conditions allowing optimum growth.

In summary, studies on the effect of the deletion of atfl-atfA-FvatfA orthologous genes on SM production gave contrasting results: loss of this bZIP-type transcription factor had either positive or negative influence on SM production, depending on the studied species and the biosynthesis route of the given SM molecule. However, in basically ROS-sensitive fungus species, like $F$. verticillioides, inactivation of $F$ vatf $A$ resulted in a significantly elevated sensitivity to ROS and this effect was accompanied with strongly retarded production of SMs, including compounds (e.g., carotenoids) with proven antioxidant activity.

The role of Atf1-AtfA-FvAtfA-type transcription factors in regulating SM production in fungi may have a great biotechnological importance. Disarmed, mycotoxin non-producing strains can be constructed for use in food and fodder industry or in biological control by gene disruption or RNAi-mediated gene-silencing technologies (Szabó et al. 2020; Damann 2014; Kagot et al. 2019). Furthermore, the manipulation of atf1-atfA-FvatfA expression may cause dramatic changes in the SM spectra of various industrial microorganisms and, therefore targeted gene expression changes may result in overproduction of highly valuable metabolites, like carotenoids or bikaverin (Szabó et al. 2020; Thomas et al. 2017).

\section{Atf1-AtfA-FvAtfA orthologs affect virulence} in pathogenic fungi

Functional analyses of atfl/atfA orthologs have been carried out in several human pathogenic fungus species, all of them classified as opportunistic pathogens.

In A. fumigatus, causing life-threatening lung and systemic infections in vulnerable, mostly immunocompromised patients, the abundantly disseminated small, stress-tolerant conidia with hydrophobic surface serve for efficient infection sources. AtfA was found to be essential for maturation of conidia and maintaining them in metabolically inactive, stress-tolerant dormancy stage, ready for dissemination and infection (Hagiwara et al. 2014, 2016); therefore, this transcription factor can be regarded as a basic component of virulence in this fungus. In another study, Silva et al. (2017) confirmed that AtfA is an important virulence factor of A. fumigatus using Galleria mellonella and neutropenic murine model of invasive pulmonary aspergillosis. Mortality rates of the $\triangle$ atfA mutant in G. mellonella were significantly lower as compared to the wild type strain. In the neutropenic murine model, the $\triangle$ atfA mutant also showed decreased virulence accompanied with weakened fungal growth (Silva et al. 2017) (Table 3).

M. circinelloides is an emerging pathogen infecting mainly immunocompromised individuals often with a fatal outcome. In this fungus, Atfl (together with Atf2) plays a key role in virulence by supporting survival of the spores after internalization by phagocytes and promoting spore germination within the phagosome (Pérez-Arques et al. 2019). The two transcription factor-encoding genes were strongly induced during macrophage phagocytosis of the wild-type fungus. Deletion of either atf 1 or atf 2 resulted in drastic reduction of virulence as tested on immunosuppressed mice. Transcriptomic experiments, performed in a mouse model, where mouse macrophages and Mucor spores were co-cultured for five $\mathrm{h}$ and then subjected to RNA sequencing, demonstrated that Atf1 and Atf2 regulate the expression of a large bulk of target genes in the intraphagosomal environment: 3384 and 3644 genes were differentially expressed in $\Delta a t f 1$ and $\Delta a t f 2$, respectively, as compared to their wild-type parent. (More than one-third of these genes were similarly regulated by the two transcription factors, suggesting an overlap between their functions.). Transcriptomic analyses revealed that Atf1 (and Atf2), besides regulating oxidative stress response, induces a variety of cellular processes including translation, carbon, and nitrogen metabolism, carotenoid biosynthesis, and cell wall biogenesis strengthening thus vigor, adaptation capability and virulence of the fungus (Table 3 ).

Deletion of atfA led to reduced virulence in P. marneffei, the cause of serious, often fatal systemic diseases in immunocompromised patients (Nimmanee et al. 2014). When mouse (J774) and human (THP1) macrophages were infected with 
Table 3 Atf1-AtfA-FvAtfA orthologs involved in the virulence of fungal species

\begin{tabular}{|c|c|c|c|}
\hline Species & Orthologs & Mechanism of action & Reference \\
\hline A. fumigatus & AtfA & $\begin{array}{l}\text { Essential for maturation of conidia } \\
\text { Increases survival in neutropenic murine } \\
\text { model and in Galleria mellonella }\end{array}$ & $\begin{array}{l}\text { Hagiwara et al. 2014, 2016; } \\
\text { Silva et al. } 2017\end{array}$ \\
\hline M. circinelloides & Atf1 & Supports survival of spores in phagocytes & Pérez-Arques et al. 2019 \\
\hline P. marneffei & AtfA & $\begin{array}{l}\text { Promotes germination of spores in phagosome } \\
\text { Increases survival of conidia in mouse and } \\
\text { macrophages }\end{array}$ & Nimmanee et al. 2014 \\
\hline C. neoformans & Atf1 & Induces the thioredoxin antioxidant system & Missall and Lodge 2005 \\
\hline $\begin{array}{l}\text { F. oxysporum } \mathrm{f} \\
\text { sp. cubense }\end{array}$ & FoAtf1 & Regulates ROS-scavenging enzymes & Qi et al. 2013 \\
\hline F. verticillioides & FvAtfA & $\begin{array}{l}\text { Increases invasive growth tested on tomato } \\
\text { fruits }\end{array}$ & Szabó et al. 2020 \\
\hline M. oryzae & MoAtf1 & Contributes to the growth of infectious hyphae & Guo et al. 2010 \\
\hline C. purpurea & CPTF1 & $\begin{array}{l}\text { Induces laccase and peroxidase enzymes } \\
\text { Contributes to normal catalase activity }\end{array}$ & Nathues et al. 2004 \\
\hline B. cinerea & BcAtf1 & Involved in conidium production & Temme et al. 2012 \\
\hline F. graminearum & FgAtf1 & Positively regulates DON production & Nguyen et al. 2013 \\
\hline
\end{tabular}

conidial suspensions, survival of the $\triangle$ atfA mutant evaluated after 24-h incubation was found to be reduced by nearly $50 \%$ as compared to the wild type. The possible reasons of this reduced virulence remained unsolved, as the in vitro assays performed on artificial medium to determine sensitivity of the mutant to oxidative stressors gave diverse results: while the $\triangle$ atfA mutant was more sensitive to $t \mathrm{BOOH}$, it showed no elevated sensitivity to hydrogen peroxide and menadione comparing with the wild type. The authors (Nimmanee et al. 2014) concluded that atf $A$ is definitely required for virulence in $P$. marneffei, but its major role in such a complex trait is other than mediating the oxidative stress response of this fungus (Table 3).

C. neoformans is a basidiomycetous yeast-causing lung infection, meningitis, and encephalitis mainly as a secondary invader of AIDS patients. (Kim et al. 2010). Pioneer studies on an atf1 ortholog of $C$. neoformans pointed out that this transcription factor-encoding gene may affect virulence, at least indirectly by regulating important elements of the oxidative stress tolerance system (Missall and Lodge 2005). Deletion of atf1 resulted in elevated sensitivity to oxidative stress of the $\Delta$ atfl strain, but the mutant displayed not much loss of virulence, as its survival rate in macrophages was similar to that of the wild-type parental strain in a mouse inhalation model. However, the thioredoxin antioxidant system of the gene deletion mutant suffered serious dysfunctions. The trx 1 gene showed delayed induction by oxidative stress and was significantly downregulated in a $\Delta a t f l$ background. Furthermore, the $\Delta$ trx 1 mutant had growth defects on artificial media and was sensitive to various stresses including oxidative stress. These findings indicate that Atfl acts as an inducer of $\operatorname{trx} 1$, an indispensable element of the thioredoxin system in the presence of oxidative stress and protects by this way the fungus from ROS-mediated defense reaction of the host macrophages (Table 3).

As a summary of these studies, atfl-atfA seems to be indispensable for virulence in opportunistic human pathogens, but a causal relationship between ROS tolerance mediated by Atfl-AtfA and virulence was difficult to find. To overcome ROS produced by host immune cells is a prerequisite for these organisms to establish disease within animal/human microenvironments, but the absence or miss-function of atfl-atfA can be circumvented by the aid of other regulators, like AtfB/Atf2 or members of the Yap1 or Skn7 families that compete with, complement, or substitute for one another. Furthermore, atfl-atfA regulates other self-defense genes required for surviv$\mathrm{al}$ in the stressful animal/human environment as demonstrated elegantly by a recent study of Pérez-Arques et al. (2019).

Among the plant pathogenic fungi subjected to functional analysis of atfl/atfA, both hemibiotrophs/biotrophs and necrotrophs occur.

Deletion of Foatfl attenuated the virulence of F. oxysporum f.sp. cubense, responsible for the Panama disease of banana (Musa spp.). The gene deletion mutant of this fungus showed elevated sensitivity to $\mathrm{H}_{2} \mathrm{O}_{2}$ and reduced catalase activity, together with downregulation of catalase-encoding genes (Qi et al. 2013) confirming that atfl is requested for virulence in biotrophic and hemibiotrophic fungi due to its regulatory role on genes encoding ROS-scavenging enzymes, like laccases, catalases, and peroxidases needed by the pathogens to impair the plant defense, mediated by reactive oxygen species (Table 3 ).

Another example of biotrophic/hemibiotrophic fungi subjected to functional analysis of the FvatfA gene is the maize pathogen $F$. verticillioides. A $\Delta F v a t f A$ mutant of this fungus also possessed increased sensitivity to oxidative 
stress-generating agents and its invasive growth, tested on tomato fruits, was significantly reduced in comparison to the wild type (Szabó et al. 2020) (Table 3).

Inefficient ROS scavenging was also found to play an important role in loss of virulence, when Moatf1, the atf1 orthologous gene of of the rice blast fungus, $M$. oryzae, was investigated. The $\Delta$ Moatfl gene deletion mutant cultivated on artificial medium had increased sensitivity to $\mathrm{H}_{2} \mathrm{O}_{2}$ and displayed markedly reduced virulence on rice plants, fully susceptible to the wild-type parental strain. Infectious hyphae of the mutant showed retarded growth, accompanied with aberrant morphological changes (Table 3). Strong defense responses, including $\mathrm{H}_{2} \mathrm{O}_{2}$ accumulation around appressoria of the fungus, were induced by the mutant at the infection site. However, when $\mathrm{H}_{2} \mathrm{O}_{2}$ generation was prevented by treating the host leaf sheaths prior to fungal inoculation with diphenyleneiodonium, a potent inhibitor of NADPH oxidase, the major source of $\mathrm{H}_{2} \mathrm{O}_{2}$ production, normal infectious growth was restored in the mutant. Transcript levels of defense-related genes of both the jasmonic acid and the salicylic acid pathways (Lox, PBZ1, AOS2 and PR1a, PAD4, $C h t 1$, respectively), as measured by qRT-PCR, were much higher in plants infected with the mutant in comparison to the wild type-infected plants. The major cause of impaired virulence of the $\Delta$ Moatfl mutant was the downregulation of laccase and peroxidase-encoding genes paralleled with a strongly reduced levels of the ROS-detoxifying enzymes encoded by these genes (Guo et al. 2010) (Table 3).

Studies on $C$. purpurea, a biotrophic pathogen of grasses and small grain cereals, demonstrated that the ATF1 homolog, CPTF1, is a general regulator of catalase activity. The $\Delta c p t f 1$ deletion mutants showed significantly weakened virulence on rye as indicated by reduced growth rate of these fungi and retarded honeydew production by the test plants at the infection site. Moreover, the rye epidermal tissues infected with $\Delta$ cptflmutants reacted with a rapid oxidative burst, whereas such response was absent in tissues infected with the wild type (Nathues et al. 2004). According to these pioneer experiments, the most likely reason of the weakened virulence found for the gene deletion mutants was their greatly impaired catalase activity. In the absence of normal catalase activity, these fungi were unable to scavenge $\mathrm{H}_{2} \mathrm{O}_{2}$ produced by the plant as a defense reaction, consequently they suffered serious ROS damage; furthermore, insufficiently scavenged $\mathrm{H}_{2} \mathrm{O}_{2}$, acting as a second messenger could efficiently alarm PTI (pathogen-triggered immunity) in the infected tissues (Table 3).

Contrarily to the situation found for biotrophic fungi, deletion of atf1-atfA-FvatfA had not much influence on ROS sensitivity in necrotrophic phytopathogens and the causes of altered virulence of the $\Delta a t f 1-\Delta a t f A-\Delta F v a t f A$ mutants of necrotrophs, if happened at all were others than ROS tolerance.

Deletion of the atfl orthologous Bcatfl gene in B. cinerea, a necrotrophic fungus with wide host range resulted in no significant change of oxidative stress sensitivity comparing with the wild type. The $\Delta B$ catfl mutant grew extremely vigorously and its colonization efficiency assessed on bean leaves and cucumber fruits was much stronger than that of the wild type (Temme et al. 2012). The aggressive colonization capability of the $\Delta B c a t f 1$ strain was of course insufficient to reach real hypervirulence, as this mutant was hampered in conidium production required for completing the disease cycle under natural conditions (Table 3). The improved colonization efficiency of $\Delta B c a t f 1$ was most probably due to its profound mycelial growth which appeared even on artificial medium, as well as the enhanced production of phytotoxins, including botrydial, botryendial, and botcinin.

$\Delta$ Fgatf1 mutants of $F$. graminearum, $\mathrm{PH}-1$ a mycotoxin-producing pathogen of maize and small grain cereals, were less sensitive to oxidative stress compared with the wild type, but they showed strongly reduced virulence on wheat and weakened colonization efficiency on maize cobs. Constitutive expression of Fgatfl resulted in hypervirulence on Brachypodium distachyon, maize and wheat, while the Fgatf1 $^{\text {oe }}$ strain remained as sensitive to oxidative stress as the wild type suggesting that atfl can enhance virulence without the improvement of ROS tolerance in this necrotrophic fungus. The $\Delta$ Fgatfl mutants produced less DON in planta by day 7 after infection than the wild type (Nguyen et al. 2013), and therefore the impaired mycotoxin production detected within plant tissues could be a reason of the attenuated virulence found for the gene deletion strains, since DON is a virulence factor due to its inhibitory effect on protein synthesis (Table 3). However, the possible role of other, still unknown factors, downregulated in the $\Delta a t f 1$ gene deletion mutant would be premature to neglect as a cause of reduced virulence. Jiang et al. (2015) studied functional relationships of three stress-related transcription factor genes, namely Fgap1, Fgatf1, and Fgskn7 of F. graminearum PH-1. (Skn7, a conserved gene in filamentous fungi is required for the upregulation of a number of oxidative stress-responsive genes.) Both $\Delta F g a t f 1$ and $\Delta F g s n k 7$ were only slightly more sensitive to $\mathrm{H}_{2} \mathrm{O}_{2}$ on complete medium than the wild type. (NB. This $\triangle F$ gatf1 strain was different from the mutants studied by Nguyen et al. (2013).). When flowering wheat heads were artificially infected with these fungi, the $\Delta$ Fgatfl mutant displayed nearly fourfold reduced disease index as compared to the wild type, whereas the $\Delta F g s n k 7$ mutant was as virulent as the wild type indicating again that deletion of atfl leads to attenuated virulence without significantly altered $\mathrm{H}_{2} \mathrm{O}_{2}$ sensitivity. DON production of $\Delta F$ gatfl was similar to the wild type on sterilized rice grain, but in infected wheat heads the mutant produced less DON than the wild type. The $\Delta F g s n k 7$ mutant produced much less DON on rice than the wild type, while its DON production in infected wheat heads was reduced only by $25 \%$ in comparison to the wild type. The $\Delta F g \operatorname{sk} 7-\Delta F g a t f 1$ double mutant was more severely 
hampered in DON production and its virulence was drastically reduced, indicating that (i) sufficient DON production rather than $\mathrm{H}_{2} \mathrm{O}_{2}$ insensitivity is an important virulence factor in $F$. graminearum, and (ii) an interplay is needed between Atf1 and other stress-related transcription factors to attain normal virulence in necrotrophic fungi.

The mode how atf1/atfA/FvatfA affects virulence is thus dependent on the lifestyle of plant pathogenic fungi. Biotrophs/hemibiotrophs require elevated ROS tolerance to achieve a successful pathogenic way of living and, therefore, Atf1-AtfA-FvAtfA, as an important element of the oxidative stress response, has a significant role in the regulation of their virulence. Contrarily, nectrotrophs need other factors for virulence (e.g., phytotoxin production, sporulation intensity, extracellular enzyme production) that may be regulated either positively or negatively by Atfl-AtfA-FvAtfA resulting in the diverse pathogenic behavior of atfl/atfA/FvatfA deletion mutants of these fungi. This conclusion is in harmony with a former paper of Heller and Tudzynski (2011), who pointed out that ROS tolerance was dispensable for virulence in necrotrophic plant pathogens.

Acknowledgements We would like to thank the valuable contribution of Ms. Ibolya Pócsi to drawing the figures.

Author contribution IP designed and ÉL, TE, KP, LH and IP wrote the manuscript.

Funding Open access fudning provided by University of Debrecen. This work was financed by the European Union and the European Social Fund through the project EFOP-3.6.1-16-2016-00022, by the National Research, Development and Innovation Office (Hungary) K119494 and NN125671 grants, and by the Thematic Excellence Programme (TKP2020-IKA-04) of the Ministry for Innovation and Technology in Hungary.

\section{Declarations}

Ethics approval This article does not contain any studies with human participants or animals performed by any of the authors.

Conflict of interest The authors declare no competing interests.

Open Access This article is licensed under a Creative Commons Attribution 4.0 International License, which permits use, sharing, adaptation, distribution and reproduction in any medium or format, as long as you give appropriate credit to the original author(s) and the source, provide a link to the Creative Commons licence, and indicate if changes were made. The images or other third party material in this article are included in the article's Creative Commons licence, unless indicated otherwise in a credit line to the material. If material is not included in the article's Creative Commons licence and your intended use is not permitted by statutory regulation or exceeds the permitted use, you will need to obtain permission directly from the copyright holder. To view a copy of this licence, visit http://creativecommons.org/licenses/by/4.0/.

\section{References}

Alves MS, Dadalto SP, Gonçalves AB, De Souza GB, Barros VA, Fietto LC (2013) Plant bZIP transcription factors responsive to pathogens: a review. Int J Mol Sci 14(4):7815-7828. https://doi.org/10.3390/ ijms 14047815

Amare MG, Keller NP (2014) Molecular mechanisms of Aspergillus flavus secondary metabolism and development. Fungal Genet Biol 66:11-18. https://doi.org/10.1016/j.fgb.2014.02.008

Antal K, Gila BC, Pócsi I, Emri T (2020) General stress response or adaptation to rapid growth in Aspergillus nidulans? Fungal Biol 124:376-386. https://doi.org/10.1016/j.funbio.2019.10.009

Avalos J, Limón MC (2015) Biological roles of fungal carotenoids. Curr Genet 61:309-324. https://doi.org/10.1007/s00294-014-0454-x

Balázs A, Pócsi I, Hamari Z, Leiter É, Emri T, Miskei M, Oláh J, Tóth V, Hegedüs N, Prade RA, Molnár M, Pócsi I (2010) AtfA BZIP-type transcription factor regulates oxidative and osmotic stress responses in Aspergillus nidulans. Mol Genet Genomics 283(3):289-303. https://doi.org/10.1007/s00438-010-0513-Z

Bruder Nascimento AC, Dos Reis TF, de Castro PA, Hori JI, Bom VL, de Assis LJ, Ramalho LN, Rocha MC, Malavazi I, Brown NA, Valiante V, Brakhage AA, Hagiwara D, Goldman GH (2016) Mitogen activated protein kinases SakA(HOG1) and MpkC collaborate for Aspergillus fumigatus virulence. Mol Microbiol 100(5): 841-859. https://doi.org/10.1111/mmi.13354

Chen D, Toone WM, Mata J, Lyne R, Burns G, Kivinen K, Brazma A, Jones N, Bähler J (2003) Global transcriptional responses of fission yeast to environmental stress. Mol Biol Cell 14(1):214-229. https:// doi.org/10.1091/mbc.e02-08-0499

Colot HV, Park G, Turner GE, Ringelberg C, Crew CM, Litvinkova L, Weiss RL, Borkovich KA, Dunlap JC (2006) A high-throughput gene knockout procedure for Neurospora reveals functions for multiple transcription factors. Proc Natl Acad Sci U S A 103:1035210357. https://doi.org/10.1073/pnas.0601456103

Damann KE (2014) Atoxigenic Aspergillus flavus biological control of aflatoxin contamination: what is the mechanism? World Mycotoxin J 8(2):235-244. https://doi.org/10.3920/WMJ2014.1719

Emri T, Szarvas V, Orosz E, Antal K, Park HS, Han K-H, Yu JH, Pócsi I (2015) Core oxidative stress response in Aspergillus nidulans. BMC Genomics 16(1):478. https://doi.org/10.1186/s12864-015-1705-Z

Emri T, Gila B, Antal K, Fekete F, Moon H, Yu JH and Pócsi I (2021) AtfA-independent adaptation to the toxic heavy metal cadmium an Aspergillus nidulans. Microorganisms, submitted

Fang Y, Xiong D, Tian L, Tang C, Wang Y, Tian C (2017) Functional characterization of two bZIP transcription factors in Verticillium dahliae. Gene 626:386-394. https://doi.org/10.1016/j.gene.2017.05.061

Fernández-Vázquez J, Vargas-Pérez I, Sansó M, Buhne K, Carmona M, Paulo E, Hermand D, Rodríguez-Gabriel M, Ayté J, Leidel S, Hidalgo E (2013) Modification of tRNA(Lys) UUU by elongator is essential for efficient translation of stress mRNAs. PLoS Genet 9 (7):e1003647. https://doi.org/10.1371/journal.pgen.1003647

Gaits F, Degols G, Shiozaki K, Russell P (1998) Phosphorylation and association with the transcription factor Atf1 regulate localization of Spc1/Sty1 stress-activated kinase in fission yeast. Genes Dev 12 (10):1464-1473. https://doi.org/10.1101/gad.12.10.1464

Gao J, Wagnon JL, Protacio RM, Glazko GV, Beggs M, Raj V, Davidson MK, Wahls WP (2013) A stress-activated, p38 mitogen-activated protein kinase-ATF/CREB pathway regulates posttranscriptional, sequence-dependent decay of target RNAs. Mol Cell Biol 33(15): 3026-3035. https://doi.org/10.1128/MCB.00349-13

Gasch AP (2007) Comparative genomics of the environmental stress response in ascomycete fungi. Yeast 24(11):961-976. https://doi. org/10.1002/yea. 1512 
Guo M, Guo W, Chen Y, Dong S, Zhang X, Zhang H, Song W, Wang W, Wang Q, Lv R, Zhang Z, Wang Y, Zheng X (2010) The basic leucine zipper transcription factor Moatfl mediates oxidative stress responses and is necessary for full virulence of the rice blast fungus Magnaporthe oryzae. MPMI 23:1053-1068. https://doi.org/10. 1094/MPMI-23-8-1053

Hagiwara D, Asano Y, Yamashino T, Mizuno T (2008) Characterization of bZip-type transcription factor AtfA with reference to stress responses of conidia of Aspergillus nidulans. Biosci Biotechnol Biochem 72(10):2756-2760. https://doi.org/10.1271/bbb.80001

Hagiwara D, Asano Y, Marui J, Yoshimi A, Mizuno T, Abe K (2009) Transcriptional profiling for Aspergillus nidulans HogA MAPK signaling pathway in response to fludioxonil and osmotic stress. Fungal Genet Biol 46:868-878. https://doi.org/10.1016/j.fgb.2009.07.003

Hagiwara D, Suzuki S, Kamei K, Gonoi T, Kawamoto S (2014) The role of AtfA and HOG MAPK pathway in stress tolerance in conidia of Aspergillus fumigatus. Fungal Genet Biol 73:138-149. https://doi. org/10.1016/j.fgb.2014.10.011

Hagiwara D, Takahashi H, Kusuya Y, Kawamoto S, Kamei K, Gonoi T (2016) Comparative transcriptome analysis revealing dormant conidia and germination associated genes in Aspergillus species: an essential role for AtfA in conidial dormancy. BMC Genomics 17, 358. https://doi.org/10.1186/s12864-016-2689-z

Heller J, Tudzynski P (2011) Reactive oxygen species in phytopathogenic fungi: signaling, development, and disease. Annu Rev Phytopathol 49:369-390. https://doi.org/10.1146/annurev-phyto072910-095355

Hirota K, Steiner WW, Shibata T, Ohta K (2007) Multiple modes of chromatin configuration at natural meiotic recombination hot spots in fission yeast. Eukaryot Cell 6(11):2072-2080. https://doi.org/10. 1128/EC.00246-07

Hong SY, Roze LV, Wee J, Linz JE (2013a) Evidence that a transcription factor regulatory network coordinates oxidative stress response and secondary metabolism in aspergilli. MicrobiologyOpen 2:144-160. https://doi.org/10.1002/mbo3.63

Hong SY, Roze LV, Linz JE (2013b) Oxidative stress-related transcription factors in the regulation of secondary metabolism. Toxins 5: 683-702. https://doi.org/10.3390/toxins5040683

Inglis DO, Binkley J, Skrzypek MS, Arnaud MB, Cerqueira GC, Shah P, Wymore F, Wortman JR, Sherlock G (2013) Comprehensive annotation of secondary metabolite biosynthetic genes and gene clusters of Aspergillus nidulans, A. fumigatus, A. niger and A. oryzae. BMC Microbiol 13:91. https://doi.org/10.1186/1471-2180-13-91

Jiang C, Zhang S, Zhang Q, Tao Y, Wang C, Xu JR (2015) FgSKN7 and FgATF1 have overlapping functions in ascosporogenesis, pathogenesis and stress responses in Fusarium graminearum. Environ Microbiol 17:1245-1260. https://doi.org/10.1111/1462-2920.12561

Jindrich K, Degnan BM (2016) The diversification of the basic leucine zipper family in eukaryotes correlates with the evolution of multicellularity. BMC Evol Biol 16:28. https://doi.org/10.1186/s12862016-0598-z

Jung M-G, Kim SS, Yu J-H, Shin KS (2016) Characterization of gprK encoding a putative hybrid G-protein-coupled receptor in Aspergillus fumigatus. PLoS One 11(9):e0161312. https://doi.org/ 10.1371/journal.pone.0161312

Kagot V, Sheila O, De Boevre M, De Saeger S (2019) Biocontrol of Aspergillus and Fusarium mycotoxins in Africa: benefits and limitations. Toxins 11(2):109. https://doi.org/10.3390/toxins 11020109

Kar P, Biswas P, Patra SK, Ghosh S (2018) Transcription factors Atfl and Sty 1 promote stress tolerance under nitrosative stress in Schizosaccharomyces pombe. Microbiol Res 206:82-90. https:// doi.org/10.1016/j.micres.2017.10.002

Kato T Jr, Okazaki K, Murakami H, Stettler S, Fantes PA, Okayama H (1996) Stress signal, mediated by a Hog1-like MAP kinase, controls sexual development in fission yeast. FEBS Lett 378(3):207-212. https://doi.org/10.1016/0014-5793(95)01442-x
Kawasaki L, Sánchez O, Shiozaki K, Aguirre J (2002) SakA MAP kinase is involved in stress signal transduction, sexual development and spore viability in Aspergillus nidulans. Mol Microbiol 45(4): 1153-1163. https://doi.org/10.1046/j.1365-2958.2002.03087.x

Kim MS, Ko Y-J, Maeng S, Floyd A, Heitman J, Bahn Y-S (2010) Comparative transcriptome analysis of the $\mathrm{CO}_{2}$ sensing pathway via differential expression of carbonic anhydrase in Cryptococcus neoformans. Genetics 185(4):1207-1219. https://doi.org/10.1534/ genetics.110.118315

Kon N, Schroeder SC, Krawchuk MD, Wahls WP (1998) Regulation of the Mts1-Mts2-dependent ade6-M26 meiotic recombination hot spot and developmental decisions by the Spc1 mitogen-activated protein kinase of fission yeast. Mol Cell Biol 18(12):7575-7583. https://doi.org/10.1128/mcb.18.12.7575

Lara-Rojas F, Sanchez O, Kawasaki L, Aguirre J (2011) Aspergillus nidulans transcription factor AtfA interacts with the MAPK SakA to regulate general stress responses, development and spore functions. Mol Microbiol 80(2):436-454. https://doi.org/10.1111/j. 1365-2958.2011.07581.x

Leong HS, Dawson K, Wirth C, Li Y, Connolly Y, Smith DL, Wilkinson CRM, Miller CJ (2014) A global non-coding RNA system modulates fission yeast protein levels in response to stress. Nat Commun 5:3947. https://doi.org/10.1038/ncomms4947

Lorenz DR, Meyer LF, Grady PJR, Meyer MM, Cam HP (2014) Heterochromatin assembly and transcriptome repression by Set 1 in coordination with a class II histone deacetylase. Elife 3:e04506. https://doi.org/10.7554/eLife.04506

Manfiolli AO, Mattos EC, de Assis LJ, Silva LP, Ulas M, Brown NA, Silva-Rocha R, Bayram Ö, Goldman GH (2019) Aspergillus fumigatus high osmolarity glycerol mitogen activated protein kinases SakA and MpkC physically interact during osmotic and cell wall stresses. Front Microbiol 10:918. https://doi.org/10.3389/ fmicb.2019.00918

Marguerat S, Lawler K, Brazma A, Bähler J (2014) Contributions of transcription an mRNA decay to gene expression dynamics of fission yeast in response to oxidative stress. RNA Biol 11(6):702-714. https://doi.org/10.4161/rna.29196

Missall TA, Lodge JK (2005) Function of the thioredoxin proteins in Cryptococcus neoformans during stress or virulence and regulation by putative transcriptional modulators. Mol Microbiol 57(3):847858. https://doi.org/10.1111/j.1365-2958.2005.04735.x

Miskei M, Karányi Z, Pócsi I (2009) Annotation of stress-response proteins in the aspergilli. Fungal Genet Biol 46(Suppl 1):S105-S120. https://doi.org/10.1016/j.fgb.2008.07.013

Nathues E, Joshi S, Tenberge KB, von den Driesch M, Oeser B, Bäumer N, Mihlan M, Tudzynski P (2004) CPTF1, a CREB-like transcription factor, is involved in the oxidative stress response in the phytopathogen Claviceps purpurea and modulates ROS level in its host Secale cereale. Mol Plant-Microbe Interact 17(4):383-393. https:// doi.org/10.1094/MPMI.2004.17.4.383

Nguyen TV, Kröger C, Bönnighausen J, Schäfer W, Jörg B (2013) The $\mathrm{ATF} / \mathrm{CREB}$ transcription factor Atfl is essential for full virulence, deoxynivalenol production, and stress tolerance in the cereal pathogen Fusarium graminearum. Mol Plant-Microbe Interact 26:13781394. https://doi.org/10.1094/MPMI-04-13-0125-R

Nimmanee P, Woo PCY, Vanittanakom P, Youngchim S, Vanittanakom $\mathrm{N}$ (2014) Functional analysis of atfA gene to stress response in pathogenic thermal dimorphic fungus Penicillium marneffei. PLoS One 9(11):e111200. https://doi.org/10.1371/journal.pone.0111200

Orosz E, Antal K, Gazdag Z, Szabó Z, Han KH, Yu JH, Pócsi I, Emri T (2017) Transcriptome-based modeling reveals that oxidative stress induces modulation of the AtfA-dependent signaling networks in Aspergillus nidulans. Int J Genomics 2017:6923849-6923814. https://doi.org/10.1155/2017/6923849

Ors A, Grimaldi M, Kimata Y, Wilkinson CR, Jones N, Yamano H (2009) The transcription factor Atfl binds and activates the APC/ 
C ubiquitin ligase in fission yeast. J Biol Chem 284(36):2398923994. https://doi.org/10.1074/jbc.M109.018309

Pérez P, Cortés JCG, Cansado J, Ribas JC (2018) Fission yeast cell wall biosynthesis and cell integrity signalling. Cell Surf 4:1-9. https:// doi.org/10.1016/j.tcsw.2018.10.001

Pérez-Arques C, Navarro-Mendoza MI, Murcia L, Lax C, MartínezGarcía P, Heitman J, Nicolás FE, Garre V (2019) Mucor circinelloides thrives inside the phagosome through an Atfmediated germination pathway. mBio 10(1):e02765-e02718. https://doi.org/10.1128/mBio.02765-18

Qi X, Guo L, Yang L, Huang J (2013) Foatf1, a bZIP transcription factor of Fusarium oxysporum f. sp. cubense is involved in pathogenesis by regulating the oxidative stress responses of Cavendish banana (Musa spp.). Physiol Mol Plant Pathol 84:76-85. https://doi.org/ 10.1016/j.pmpp.2013.07.007

Reverberi M, Zjalic S, Ricelli A, Punelli F, Camera E, Fabbri C, Picardo M, Fanelli C, Fabbri AA (2008) Modulation of antioxidant defense in Aspergillus parasiticus is involved in aflatoxin biosynthesis: a role for the ApyapA gene. Eukaryot Cell 7(6):988-1000. https:// doi.org/10.1128/EC.00228-07

Reverberi M, Gazzetti K, Punelli F, Scarpari M, Zjalic S, Ricelli A, Fabbri AA, Fanelli C (2012) Aoyap1 regulates OTA synthesis by controlling cell redox balance in Aspergillus ochraceus. Appl Microbiol Biotechnol 95(5):1293-1304. https://doi.org/10.1007/s00253-012-3985-4

Rodrigues-Pousada C, Menezes RA, Pimentel C (2010) The Yap family and its role in stress response. Yeast 27(5):245-258. https://doi.org/ $10.1002 /$ yea. 1752

Roze LV, Chanda A, Wee J, Awad D, Linz JE (2011) Stress-related transcription factor AtfB integrates secondary metabolism with oxidative stress response in aspergilli. J Biol Chem 286:35137-35148. https://doi.org/10.1074/jbc.M111.253468

Sakamoto K, Arima TH, Iwashita K, Yamada O, Gomi K, Akita O (2008) Aspergillus oryzae atfB encodes a transcription factor required for stress tolerance in conidia. Fungal Genet Biol 45(6):922-932. https://doi.org/10.1016/j.fgb.2008.03.009

Sakamoto K, Iwashita K, Yamada O, Kobayashi K, Mizuno A, Akita O, Mikami S, Shimoi H, Gomi K (2009) Aspergillus oryzae atfA controls conidial germination and stress tolerance. Fungal Genet Biol 46 (12):887-897. https://doi.org/10.1016/j.fgb.2009.09.004

Salat-Canela C, Paulo E, Sánchez-Mir L, Carmona M, Ayté J, Oliva B, Hidalgo E (2017) Deciphering the role of the signal- and Sty1 kinase-dependent phosphorylation of the stress-responsive transcription factor Atfl on gene activation. J Biol Chem 292(33): 13635-13644. https://doi.org/10.1074/jbc.M117.794339

Sánchez-Mir L, Salat-Canela C, Paulo E, Carmona M, Ayté J, Oliva B, Hidalgo E (2018) Phospho-mimicking Atfl mutants bypass the transcription activating function of the MAP kinase Sty1 of fission yeast. Curr Genet 64(1):97-102. https://doi.org/10.1007/s00294017-0730-7

Sansó M, Gogol M, Ayté J, Seidel C, Hidalgo E (2008) Transcription factors Pcr1 and Atf1 have distinct roles in stress- and Sty1dependent gene regulation. Eukaryot Cell 7:826-835. https://doi. org/10.1128/EC.00465-07

Sansó M, Vargas-Pérez I, García P, Ayté J, Hidalgo E (2011) Nuclear roles and regulation of chromatin structure by the stress-dependent MAP kinase Sty1 of Schizosaccharomyces pombe. Mol Microbiol 82(3):542-554. https://doi.org/10.1111/j.1365-2958.2011.07851.x

Shiozaki K, Russell P (1996) Conjugation, meiosis, and the osmotic stress response are regulated by Spc1 kinase through Atf1 transcription factor in fission yeast. Genes Dev 10(18):2276-2288. https:// doi.org/10.1101/gad.10.18.2276

Silva LP, Alves de Castro P, Dos Reis TF, Paziani MH, Von Zeska Kress MR, Riaño-Pachón DM, Hagiwara D, Ries LN, Brown NA, Goldman GH (2017) Genome-wide transcriptome analysis of Aspergillus fumigatus exposed to osmotic stress reveals regulators of osmotic and cell wall stresses that are SakA ${ }^{\mathrm{HOG} 1}$ and $\mathrm{MpkC}$ dependent. Cell Microbiol 19(4). https://doi.org/10.1111/cmi.12681

Silva LP, Horta MAC, Goldman GH (2021) Genetic interactions between Aspergillus fumigatus basic leucine zipper (bZIP) transcription factors AtfA, AtfB, AtfC and AtfD. Frontiers In Fungal Biol 2:632048. https://doi.org/10.3389/ffunb.2021.632048

Steiner WW, Schreckhise RW, Smith GR (2002) Meiotic DNA breaks at the $S$. pombe recombination hot spot M26. Mol Cell 9(4):847-855. https://doi.org/10.1016/s1097-2765(02)00489-6

Szabó Z, Pákozdi K, Murvai K, Pusztahelyi T, Kecskeméti Á, Gáspár A, Logrieco AF, Emri T, Ádám AL, Leiter É, Hornok L, Pócsi I (2020) FvatfA regulates growth, stress tolerance as well as mycotoxin and pigment productions in Fusarium verticillioides. Appl Microbiol Biotechnol 104(18):7879-7899. https://doi.org/10.1007/s00253-020-10717-6

Takada H, Nishimura M, Asayama Y, Mannse Y, Ishiwata S, Kita A, Doi A, Nishida A, Kai N, Moriuchi S, Tohda H, Giga-Hama Y, Kuno T, Sugiura R (2007) Atfl is a target of the mitogen-activated protein kinase Pmk1 and regulates cell integrity in fission yeast. Mol Biol Cell 18(12):4794-4802. https://doi.org/10.1091/mbc.e07-03-0282

Takeda T, Toda T, Kominami K, Kohnosu A, Yanagida M, Jones N (1995) Schizosaccharomyces pombe atfl ${ }^{+}$encodes a transcription factor required for sexual development and entry into stationary phase. EMBO J 14(24):6193-6208. https://doi.org/10.1002/j.14602075.1995.tb00310.x

Temme N, Oeser B, Massaroli M, Heller J, Simon A, Collado IG, Viaud M, Tudzynski P (2012) BcAtfl, a global regulator, controls various differentiation processes and phytotoxin production in Botrytis cinerea. Mol Plant Pathol 13:704-718. https://doi.org/10.1111/j. 1364-3703.2011.00778.x

Thomas AB, Shetane TD, Singha RG, Nanda RK, Poddar SS, Shirsat A (2017) Employing central composite design for evaluation of biomass production by Fusarium venenatum: In vivo antioxidant and antihyperlipidemic properties. Appl Biochem Biotechnol 183(1): 91-109. https://doi.org/10.1007/s12010-017-2432-5

Tomee JF, Kauffman HF (2000) Putative virulence factors of Aspergillus fumigatus. Clin Exp Allergy 30(4):476-484. https://doi.org/10. 1046/j.1365-2222.2000.00796.x

Yamashita K, Shiozawa A, Watanabe S, Fukumori F, Kimura M, Fujimura M (2008) ATF-1 transcription factor regulates the expression of ccg-1 and cat-1 genes in response to fludioxonil under OS-2 MAP kinase in Neurospora crassa. Fungal Genet Biol 45(12): 1562-1569. https://doi.org/10.1016/j.fgb.2008.09.012

Yin WB, Amaike S, Wohlbach DJ, Gasch AP, Chiang YM, Wang CC, Bok JW, Rohlfs M, Keller NP (2012) An Aspergillus nidulans bZIP response pathway hardwired for defensive secondary metabolism operates through aflR. Mol Microbiol 83:1024-1034. https://doi. org/10.1111/j.1365-2958.2012.07986.x

Valero C, Colabardini AC, Chiaratto J, Pardeshi L, de Castro PA, Filho JAF, Silva LP, Rocha MC, Malavazi I, Costa JH, Fill T, Barros MH, Wong SSZW, Aimanianda V, Wong KH, Goldman GH (2020) Aspergillus fumigatus transcription factors involved in the caspofungin paradoxical effect. 11(3):mBio, e00816-e0mB20. https://oi.org/10.1128/mBio.00816-20

Valiante V, Macheleidt J, Föge M, Brakhage AA (2015) The Aspergillus fumigatus cell wall integrity signaling pathway: drug target, compensatory pathways, and virulence. Front Microbiol 6:325. https:// doi.org/10.3389/fmicb.2015.00325

Watson G, Ronai ZA, Lau E (2017) ATF2, a paradigm of the multifaceted regulation of transcription factors in biology and disease. Pharmacol Res 119:347-357. https://doi.org/10.1016/j.phrs.2017. 02.004

Publisher's note Springer Nature remains neutral with regard to jurisdictional claims in published maps and institutional affiliations. 